\title{
Consider Differentials before Diagnosing AMSAN in COVID-19 Patients
}

\author{
Josef Finsterer, MD, $\mathrm{PhD}^{1 *}$; Fulvio Alexandre Scorza, $\mathrm{MD}^{2}$; Carla Alessandra Scorza, $\mathrm{MD}^{2}$; Ana Claudia Fiorini, $\mathrm{MD}^{3}$
}

'Municipial Hospital Landstrasse, Messerli Research Institute, Vienna, Austria

${ }^{2}$ Dptartment of Neuroscience, University of São Paulo (UNIFESP/EPM), São Paulo, Brazil

${ }^{3}$ University of Sao Paolo, São Paolo (PUC-SP), Brazil

$\mathrm{W}$ th interest, we read the article by Agha Abbaslou et al about a 55-year-old female with COVID-19 who developed the axonal type of Guillain-Barre syndrome (GBS), known as acute motor and sensory axonal neuropathy (AMSAN) 26 days after becoming symptomatic with the viral infection. ${ }^{1}$ It was concluded that care providers should be aware of newly developing neurological abnormalities in COVID-19 patients, particularly those hospitalised for the infection. ${ }^{1}$ We have the following comments and concerns.

The main shortcoming of the study is that the diagnosis of AMSAN remains speculative. Arguments against AMSAN are that only the lower limbs were clinically affected, that protein in the cerebrospinal fluid (CSF) was normal, and that immunoglobulins were ineffective. Further arguments against a causal relation between COVID-19 and the neuropathy are that neuropathy developed 26 days after the onset of the infection and that viral RNA was not confirmed in the CSF.

The presented patient had chronic lung disease of unknown etiology. ${ }^{1}$ Thus, it can be expected that the patient was regularly taking drugs. We should be informed about the current medication the patient was regularly taking. Knowing the current medication is crucial as it may contribute to the development of complications and thus, may have determined the outcome of this patient. Patients with chronic lung disease frequently take longterm corticosteroids; therefore, we should know if the patient had developed steroid myopathy. We also should know if the patient was taking any medication with neurotoxic side effects which could be responsible for the findings in nerve conduction studies.

Lopinavir and ritonavir have been reported to be neurotoxic and may occasionally cause neuropathy. ${ }^{2}$ We should know if the authors considered these compounds responsible for the abnormalities in nerve conduction studies. The patient had received these compounds since 11 days prior to the onset of muscle weakness. ${ }^{1}$ Since the patient received neurotoxic drugs and was treated in the ICU for 20 days, critical neuropathy is the much more likely diagnosis in the presented patient than AMSAN.

Though GBS is rarely associated with COVID-19, a recent review reported 62 patients with severe acute respiratory syndrome coronavirus 2 (SARS-CoV-2) associated GBS. ${ }^{3}$ Among these patients, 42 were diagnosed with acute inflammatory demyelinating polyneuropathy (AIDP), 6 with acute, motor axonal neuropathy, 5 with Miller-Fisher syndrome, and 3 with AMSAN. ${ }^{3}$ We thus do not agree with the statement that AMSAN is a rare subtype of GBS. ${ }^{1}$ AMSAN may be rare in the Western world but is more prevalent than AIDP in Asia. ${ }^{4}$ It would be interesting to know the prevalence of AMSAN and AIDP in Iran.

We do not agree with the statement that Zhao et al reported the one and only patient with SARS-CoV-2 associated GBS in whom polyradiculitis was the presenting manifestation. ${ }^{5}$ Among the 62 patients with SARS-CoV-2 associated GBS mentioned above, 4 presented with GBS before the onset of clinical manifestations of the viral infection. ${ }^{3}$

In the vast majority of cases, the CSF is negative for viral RNA in SARS-CoV-2 associated GBS. ${ }^{3}$ However, some patients may be positive for the viral RNA although it is unknown if those positive for the virus in the CSF have a worse outcome than those negative for the virus. Patients positive for the virus in the CSF may also have encephalitis, ${ }^{6}$ which may strongly determine the outcome. We should know why the patient was discharged from the ICU despite persisting respiratory distress. Since GBS may be associated with Bickerstaff encephalitis, we should know the results of cerebral imaging with contrast medium.

Overall, the interesting report has a number of shortcomings, which should be addressed before drawing 
final conclusions. The diagnosis of AMSAN should be discussed, the current medication and cerebral MRI should be reported, and the CSF should be investigated for viral RNA. It should be stressed that AMSAN has been previously reported in association with COVID-19 and that several other patients develop clinical manifestations of GBS prior to the onset of the viral infection.

\section{Authors' Contribution}

All authors contributed equally.

\section{Conflict of Interest Disclosures}

The authors declare no conflicts of interest.

\section{Ethical Statement}

Not applicable.

\section{References}

1. Agha Abbaslou M, Karbasi M, Mozhdehipanah H. A Rare
Axonal Variant of Guillain-Barré Syndrome as a Neurological Complication of COVID-19 Infection. Arch Iran Med. 2020;23:718-21. doi: 10.34172/aim.2020.93.

2. Khanlou H,Valdes-Sueiras M, Farthing C. Peripheral Neuropathy Induced by Lopinavir-Saquinavir-Ritonavir Combination Therapy in an HIV-Infected Patient. J Int Assoc Physicians AIDS Care. 2007;6:155. doi: 10.1177/1545109707302756.

3. Finsterer J, Scorza FA, Fiorini AC. SARS-CoV-2-associated Guillain-Barre syndrome in 62 patients. Eur J Neurol. 2020;25:10.1111/ene.14544. doi: 10.1111/ene.14544.

4. Vucic S, Kiernan MC, Cornblath DR. Guillain-Barré syndrome: an update. J Clin Neurosci. 2009;16(6):733-41. doi: 10.1016/j. jocn.2008.08.033.

5. Zhao H, Shen D, Zhou H, Liu J, Chen S. Guillain-Barré syndrome associated with SARS-CoV-2 infection: causality or coincidence?. Lancet Neurol. 2020;19:383-384. doi: 10.1016/S1474-4422(20)30109-5.

6. Dalakas MC. Guillain-Barré syndrome: The first documented COVID-19-triggered autoimmune neurologic disease: More to come with myositis in the offing. Neurol Neuroimmunol Neuroinflamm. 2020;7:e781. doi: 10.1212/ NXI.0000000000000781. 